\title{
A comparison of poly-ethylene-glycol-coated and uncoated gold nanoparticle-mediated hepatotoxicity and oxidative stress in Sprague Dawley rats
}

This article was published in the following Dove Medical Press journal:

International Journal of Nanomedicine

\begin{abstract}
Anita K Patlolla ${ }^{1,2}$
S Anitha Kumari ${ }^{3}$

Paul B Tchounwou ${ }^{1,2}$

'NIH-RCMI Center for Environmental Health, College of Science Engineering and Technology, Jackson State University, Jackson, MS, 39217, USA; ${ }^{2}$ Department of Biology, CSET, Jackson State University, Jackson, MS, 39217, USA; ${ }^{3}$ Department of Zoology, University College for Women, Koti, Hyderabad, Telangana, India
\end{abstract}

Video abstract

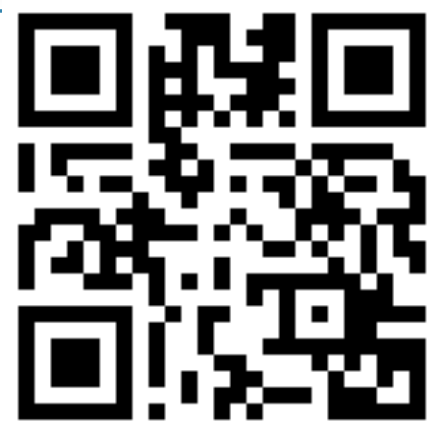

Point your SmartPhone at the code above. If you have a QR code reader the video abstract will appear. Or use:

http://youtu.be/ L2uRVTVhQU
Correspondence: Anita K Patlolla NIH-RCMI Center for Environmental Health, College of Science Engineering and Technology, Jackson State University, 1400 Lynch Street, Jackson, MS-39217, USA Tel + l 60I 9790210

Fax + I 60I 9795853

Email anita.k.patlolla@jsums.edu
Background: Gold nanoparticles (GNPs) and their functional derivatives are of great interest because of their many biomedical applications. GNPs are increasingly being incorporated into new diagnostic and therapeutic approaches in medicine. Consequently, there has been a strong push to fully understand their interactions with blood components. The agglomeration of cells reflects the interaction of nanoparticles with blood components.

Methods: The main aim of this study was to compare the effects of poly-ethylene-glycol (PEG)-oated and uncoated GNPs on the generation of reactive oxygen species (ROS); on the actions of distinct hepatotoxicity biomarkers such as alanine (ALT) and aspartate (AST) aminotransferases, and alkaline phosphatase (ALP); and on the histology of liver tissues in the rat model. Four distinct doses of PEG-coated and uncoated GNPs $(12.5,25,50$, and $100 \mu \mathrm{g} / \mathrm{kg}$ body weight) were used. Each group consisted of three rats receiving an oral administration of PEG-coated and uncoated GNPs for 5 days with one dose per 24 hours. The control group consisted of three rats that received deionized water. Twenty-four hours after the last treatment, samples were collected following standard procedures.

Results: PEG-coated and uncoated GNPs enhanced the generation of ROS and the activity of serum aminotransferases (ALT/AST) and ALPs relative to the negative control. A liver histology assessment of GNP-exposed rats revealed statistically significant responses in the variation of the morphologies of tissues relative to those of the negative control. Nonetheless, uncoated GNPs demonstrated enhanced hepatotoxic outcomes relative to those of PEG-coated GNPs. The results demonstrated that both GNPs may be able to promote hepatotoxicity in Sprague Dawley rats through mechanisms of oxidative stress. However, uncoated GNPs have more harmful effects than PEG-coated GNPs relative to the negative control.

Conclusion: Taken together, the results of this study indicate that PEG-coated GNPs may be safer to use in nanomedicinal applications than uncoated GNPs. However, more studies must be performed to confirm the outcomes of PEGylation.

Keywords: serum aminotransferases, polyethylene glycol coated gold nanoparticles, uncoated gold nanoparticles, hepatotoxicity, oxidative stress

\section{Introduction}

Gold-based nanoparticles (GNPs) have been widely used for various biomedical purposes (eg, biosensors, optical imaging, drug-delivery, and photothermal therapy). These uses of GNPs are made possible due to their surface chemistry and optical characteristics. In considering these uses, the biocompatibility and non-toxic nature 
of GNPs are vital. Once GNPs enter the body, they can be translocated through the blood to accessory target organs such as the liver, spurring adverse biological reactions. ${ }^{1}$

Despite their significant potential environmental, biomedical, and industrial applications, there are very limited data on corresponding acute and chronic health responses in humans and the environment. Consequently, there are no safety and regulatory guidelines concerning the manufacture and application of nanomaterials. ${ }^{2}$

The in vivo biocompatibility of GNP has been widely investigated. Concerns regarding GNP blood compatibility have been raised due to its increased use in biomedical applications. Some attributes that have been closely related to the toxicity of GNPs include their surface chemistry, physical dimensions, doses, and routes of administration. Modifications of GNPs with different functional groups such as citrate, tannic acid, polyvinylpyrrolidone, polyvinyl alcohol, silica, and amine-terminated silica including polyethylene glycol (PEG) have been shown to alter cell-particle interactions, ensuring system stability and biocompatibility. Several studies $^{3,4}$ have reported that uncoated GNPs are unstable under physiological conditions and are able to agglomerate in blood through interactions with proteins. These agglomerates have been shown to reach the liver and spleen via rapid absorption through the reticuloendothelial system. (PEG)-coated GNP has been found to reduce surface charges (zeta potential) and improve the blood stability of GNPs. ${ }^{5}$ In addition, they are found in monodisperse distributions under physiological conditions with an extensive half-life; thus, they are constantly found in circulating blood. Despite these positive qualities, PEG-coated GNPs are still capable of being toxic to the liver and kidneys and to spurring gene expression from accumulation in these tissues.

As GNPs accumulate in these tissues, they have been shown to produce reactive oxygen species (ROS) and to mainly cause oxidative stress when the chemical reaction conditions of cells are overloaded. ${ }^{6-9}$ ROS generation is a normal physiological process implicated in various forms of cellular signaling, including defense mechanisms of the immune system. However, in surplus it can cause serious injury to cellular macromolecules such as proteins, lipids, and DNA. The quantity of ROS generated by GNPs has been shown to be a function of diameter and surface area. The smaller the diameter and larger the surface area, the more the ROS that is generated. GNPs of 5-250 nm have disclosed an inverse association between smaller diameter and larger surface area nanoparticles in generating elevated quantities of ROS. ${ }^{10}$ In spite of this, the relationship between GNP and oxidative stress is not well understood. ${ }^{11}$

The liver is a vital organ that is essential to sustaining life in many organisms due to its numerous functions. Most notably, the liver produces proteins including those involved in blood clotting. The liver is also responsible for the detoxification of blood and for the metabolism of nutrients from the digestive tract. Consequently, the impairment of liver function can have very serious effects. ${ }^{12}$ There are multiple means of measuring hepatotoxicity. Alanine (ALT) and aspartate (AST) aminotransferases are considered to be markers of direct cellular injury to hepatocytes. ALT is considered a more specific marker than AST. ${ }^{1,12}$ Alkaline phosphatase (ALP) is another marker correlated with cholestatic or congestive problems in the liver. These problems can either occur secondary to liver injury or can progress to liver injury due to obstruction. Measuring the levels of such enzymes in the blood has become a widely used approach for assessing hepatotoxicity over the past 25 years. ${ }^{13}$

This study assesses the effects, after oral administration of PEG-coated and uncoated GNPs, on ROS induction, on levels of various hepatotoxicity biomarkers (ALT/AST; ALP), and on histopathology changes of liver tissues in the rat model. The question of the health effects of GNPs is quite acute, and this study brings new data in a field where the largest proportion of publications have been conducted with non-comparative models. The few studies involving oral delivery of GNPs focus on the possible oral absorption in gastrointestinal tract, or on pharmacokinetics. The liver is one of the recurrent target organs found after oral, intravenous (iv), or intraperitoneal (ip) injection of GNPs in diverse publications and even sometimes the dominant site of accumulation. Therefore, the results presented here are of importance for health risk assessment.

\section{Materials and methods Chemicals and reagents}

PEG-coated and uncoated GNPs in liquid form (25 nm) were purchased from Sigma-Aldrich Co. (St Louis, MO, USA). Xylene, ethyl alcohol, paraffin wax, H\&E stain, and diagnostic enzyme assay kits were acquired from SigmaAldrich. A DCFH-DA assay kit was purchased from Abcam (Cambridge, UK).

\section{Animals}

From Harlan Sprague Dawley Breeding Laboratories in Indianapolis, IN, USA, we acquired healthy adult male 
Sprague Dawley rats (6-8 weeks of age, with an average body weight (BW) of $100 \pm 2 \mathrm{~g}$ ). Animals were allowed to adapt to the animal housing for 1 week prior to the experiments. The rats were indiscriminately chosen and accommodated in polycarbonate cages $(18.88$ in $\times 7.25$ in $\times 3.76$ in) (three rats per cage) with steel wire tops and corn-cob bedding. They were retained in a supervised atmosphere with a 12-hour dark/12-hour light cycle, a temperature of $22^{\circ} \mathrm{C} \pm 2^{\circ} \mathrm{C}$, and humidity levels of $50 \%-70 \%$. Free access to food (standard rat pellets, Sniff [oval normal diet of balanced nutritional value for biomedical research]) and fresh tap water was made available to the rats.

The animal protocols used in this work were evaluated and approved by the Animal Use and Ethic Committee (CEUA) of the 0804 (Ethical Approval Code). They are in accordance with FELASA guidelines and with the National Law for Laboratory Animal Experimentation (Law no 18.611). ${ }^{28}$

\section{Experimental design}

Four distinct doses of PEG-coated and uncoated GNPs $(12.5,25,50$, and $100 \mu \mathrm{g} / \mathrm{kg} \mathrm{BW})$ were used. Each group consisted of three rats receiving oral administrations of PEG-coated and uncoated GNPs, given for 5 days at one dose per 24 hours. The control group included three rats that received deionized water. All experiments were regulated in compliance with the National and Institutional guidelines for the care and use of animals in biomedical research. ${ }^{14}$

To determine the morphological size of PEG-coated and uncoated GNPs (Figure 1), a transmission electron microscope (TEM) was utilized. A drop of homogeneously dispersed GNP was suspended on a copper grid with lacey carbon film and was allowed to air dry. The images of both types of GNPs were captured using a field emission JEOL-JEM2100F, TEM, operating at $200 \mathrm{KV}$ (JEOL, Tokyo, Japan).

To determine the size and zeta potential of PEG-coated and uncoated GNPs, a Nano Zetasizer (Malvern Instruments,
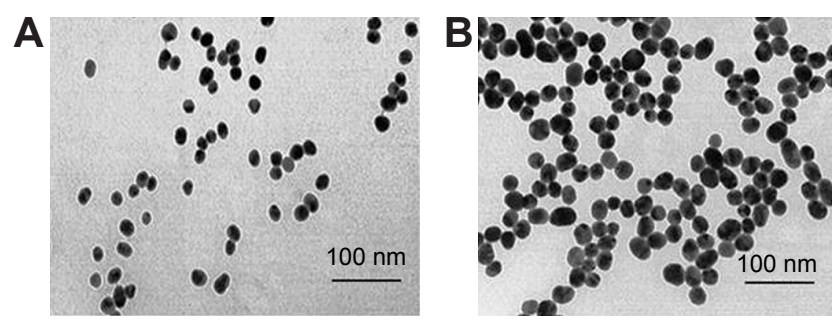

Figure I Transmission electron microscope structures of $(\mathbf{A})$ uncoated GNP and (B) PEG-coated GNP.

Abbreviations: GNPs, gold nanoparticles; PEG, poly-ethylene-glycol.
Malvern, UK) was used. To obtain a homogenous dispersion of nanoparticles, a stock solution of $50 \mu \mathrm{g} / \mathrm{mL}$ of GNPs was diluted, vortexed, and sonicated for 5 minutes. One milliliter of the diluted GNPs dispersion was transferred to a $1 \mathrm{~cm}$ cuvette for dynamic size measurements of both GNPs. A Malvern zeta potential cell was utilized to measure zeta potential values. The cell was rinsed 3-4 times with ultrapure water before transferring $850 \mu \mathrm{L}$ of the diluted GNPs dispersion into it. GNPs were used as a positive control to authenticate the instrument. Each measurement was collected three times and specifics acquired were determined as the average size or zeta potential of GNPs.

\section{Liver homogenate preparation}

Livers exposed to PEG-coated and uncoated GNPs were excised under anesthesia after 5 days of exposure. A $10 \%$ homogenate of liver tissue was formulated in a solution of $0.05 \mathrm{M}$ phosphate buffer ( $\mathrm{pH}$ 7.4) and $0.01 \mathrm{mM}$ EDTA. The liver tissue was homogenized using a motor-driven Teflonpestle homogenizer (Thermo Fisher Scientific, Waltham, MA, USA), followed by sonication (Branson Sonifier) and centrifugation at $500 \times \mathrm{g}$ for 10 minutes at $4^{\circ} \mathrm{C}$. The supernatant was transferred and centrifuged at $2,000 \times g$ for 60 minutes at $4^{\circ} \mathrm{C}$. Following centrifugation, cellular fragments were collected and utilized as homogenate for assessment.

\section{Detection of ROS}

The manufacturer's protocol for DCFH-DA ( $\left(2^{\prime} 7^{\prime}\right.$ dichlorofluorescin diacetate) with slight modifications was followed to analyze ROS production. ${ }^{15}$ Samples were measured using a Fluorescence Plate Reader (Turner Biosystems, Sunnyvale, CA, USA) for which peak excitation wavelengths for oxidized DCFH were set at $488 \mathrm{~nm}$ and emission wavelengths at $525 \mathrm{~nm}$. The calibration of the fluorometry process was executed using standard curves for serial dilutions of fluorescein. The samples underwent serial dilutions of metal-contaminant free hydrogen peroxide (Merck, Darmstadt, Germany) and were incubated. The oxidation of DCFH was then measured per $\mu \mathrm{M} \mathrm{H}_{2} \mathrm{O}_{2}$. Samples loaded with DCFH-DA were deducted by their appropriate controls to measure accurate DCFH oxidation levels of the liver samples.

\section{Collection of serum}

Samples of blood were collected in heparin containing tubes 24 hours after the last treatment following normal protocols. A sample of blood from each group was allowed to clot 
for 30 minutes, followed by centrifugation $(750 \times g$ for 15 minutes). Serum was drained from cellular elements and transferred to an acid-washed polypropylene tube properly labeled and stored until further assessment. Colorimetric assay kits were utilized to measure levels of alanine (ALA) and aspartate (ASP) aminotransferases, and alkaline phosphatase (ALP) in the serum.

\section{Serum aminotransferases}

Methods developed by Reitman and Franke ${ }^{16}$ were used to measure alanine or glutamate pyruvate transaminase (ALT/ GPT) and aspartate or glutamate oxaloacetate transaminase (AST/GOT) levels in the serum. While several types of transaminases are present in human serum, ALT/GPT and AST/GOT are the two most frequently measured enzymes. The mechanism involved in the activities of these enzymes involves the catalyzed transfer of alpha-amino groups from specific amino acids to alpha-ketoglutaaric acid (AKG) to yield respective end products glutamic and oxaloacetice acid. 2, 4-dinitrophenyl hydrazine reactivity with keto acids was detected using a colorimetric assay kit. At a wavelength of $\sim 505 \mathrm{~nm}$, the absorbance of the resulting color was determined to have beneficial effects on absorption between hydrazones of AKG and hydrazones of oxaloacetic or pyruvic acid.

\section{Analysis of alkaline phosphatases}

A method developed by Kay et a $1{ }^{17}$ was employed to evaluate the activity of ALP in serum using a diagnostic kit developed by Sigma-Aldrich Co. The mechanism involved in relies on the hydrolysis of p-nitrophenyl phosphate by the enzyme, generating p-nitrophenol and inorganic phosphate. When made alkaline, p-nitrophenol is converted into a yellow complex that is readily measured at a wavelength of $400-420 \mathrm{~nm}$. Phosphatase activity has been shown to be directly proportional to the intensity of color formed.

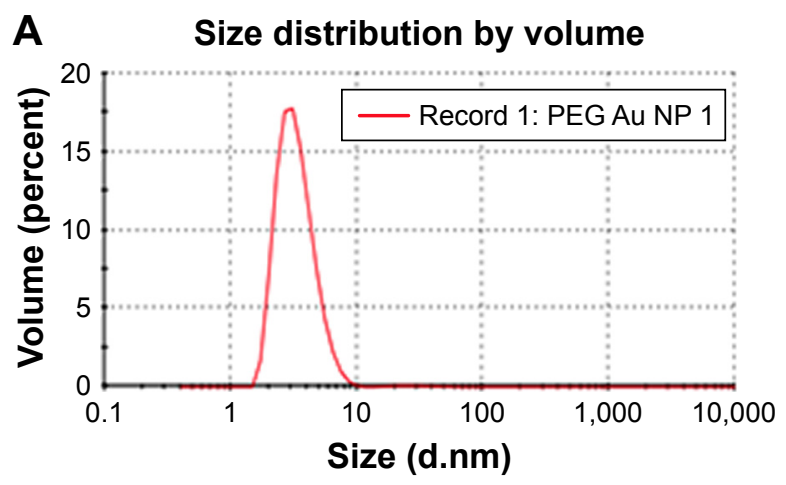

Figure 2 Gold nanoparticle sizes measured in deionized water using a Nano Zetasizer. Note: (A) PEG-coated and (B) uncoated by dynamic light scattering intensity. Abbreviations: PEG, poly-ethylene-glycol; NP, nanoparticle.

\section{Histology of liver}

For morphological analysis, liver (same lobe) tissues were isolated from control and exposed rats under anesthesia, were rinsed with ice-cold normal saline $(0.9 \% \mathrm{NaCl})$ and $20 \mathrm{mM}$ EDTA to remove blood, were fixed using $10 \%$ formalin for 48 hours, and were embedded in paraffin. The liver sections were stained using H\&E. The percentage of hepatic pathological alterations was evaluated via semiquantitative estimation. Ten slides of each sample were scored for liver histology. The following criteria were used to score liver morphologies: $0=$ no hepatocyte damage (normal), 1 = mild hepatocyte damage, 2 = moderate hepatocyte damage, 3 = extensive hepatocyte damage.

\section{Statistical analysis}

A statistical analysis was performed with SAS 9.1 software for Windows XP. Mean \pm SDs were presented as data. Oneway ANOVA $P$-values of $<0.05$ were considered statistically significant. Dunnett's $t$-test was used for the post hoc evaluation of data.

\section{Results \\ Nanomaterial characterization}

PEG-coated and uncoated GNPs were characterized using a TEM (JEOL-1011) (Figure 1) to understand morphologies, diameters, tendencies of aggregation, and cellular distributions. Dynamic light scattering (DLS) was employed for both GNP samples (Figure 2A and B) to study the dispersion of particles when placed into deionized water. According to results obtained through the DLS and TEM analysis of aqueous solution, it was determined that both particles range in size from 15 to $35 \mathrm{~nm}$ and that the particles are spherically shaped. Zeta potential values of the PEG-coated and uncoated GNPs were found to be $-14.5 \mathrm{mV}$ and $54.3 \mathrm{mV}$, respectively.

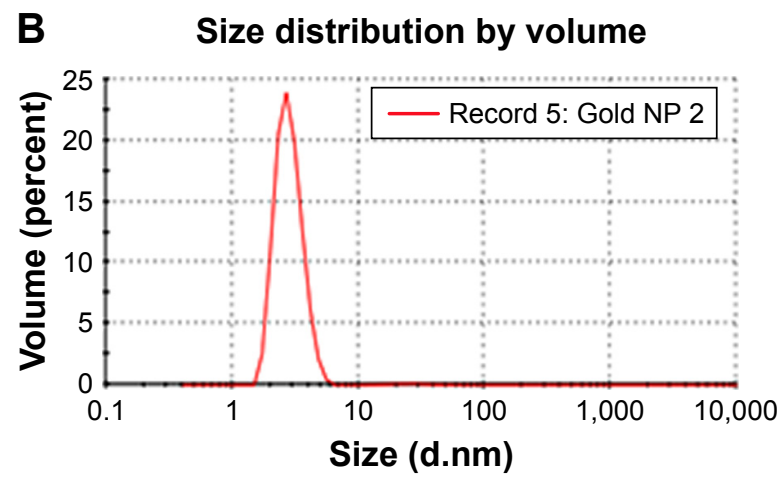




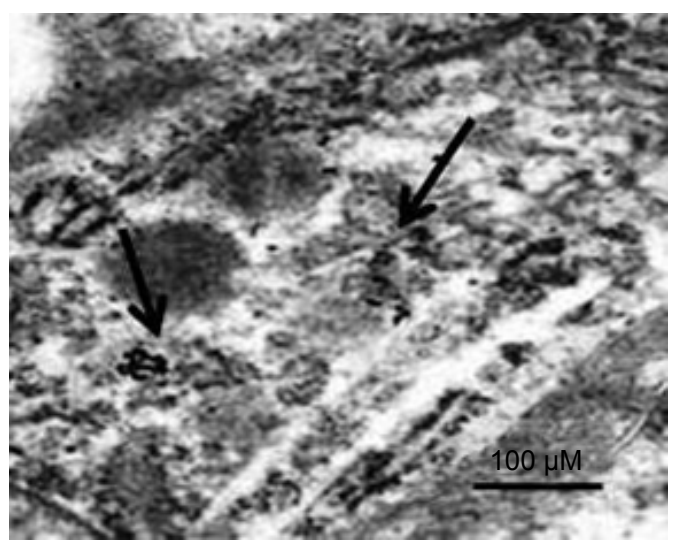

Figure 3 TEM image of the uptake of gold nanoparticles in the livers of rats. Note: Black arrows indicate where there is accumulation of gold nanoparticles. Abbreviation: TEM, transmission electron microscope.

\section{Distribution of GNPs in rat livers}

To determine the localization of GNPs in the livers of rats after 5 days of exposure, a TEM analysis was conducted. GNPs were predominantly found in Kupffer cells of the liver. Figure 3 presents a photomicrograph of the uptake of GNP.

\section{ROS detection}

PEG-coated and uncoated GNPs elevated the induction of ROS in the exposed rats relative to the negative control. The highest two doses, 50 and $100 \mu \mathrm{g} / \mathrm{kg}$, produced a statistically remarkable difference in the generation of ROS in both types of GNPs relative to the negative control. Experimental data on the intracellular generation of ROS in rats exposed to PEG-coated and uncoated GNPs and for the negative control are shown in Figure 4. However, more ROS production was

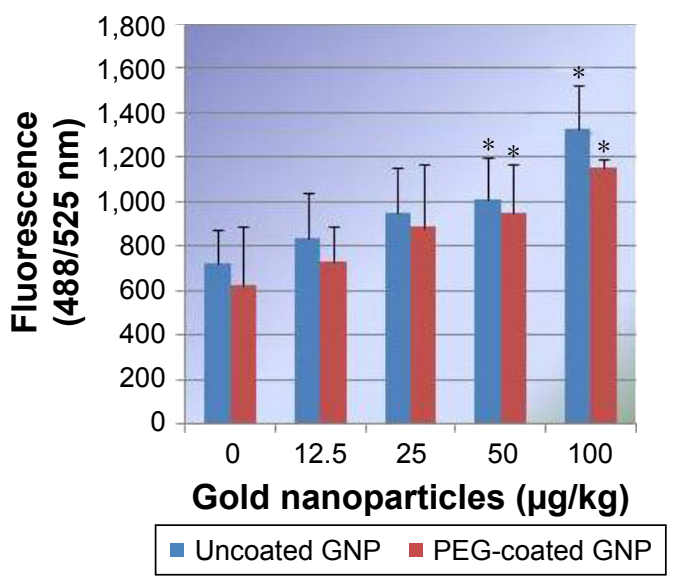

Figure 4 Effects of PEG-coated and uncoated gold nanoparticles on the generation of reactive oxygen species in Sprague Dawley rats.

Notes: The mean \pm SD of three rats represent each bar. Values shown with asterisks are significantly different from the control; $* P<0.05$.

Abbreviations: GNP, gold nanoparticle; PEG, poly-ethylene-glycol.

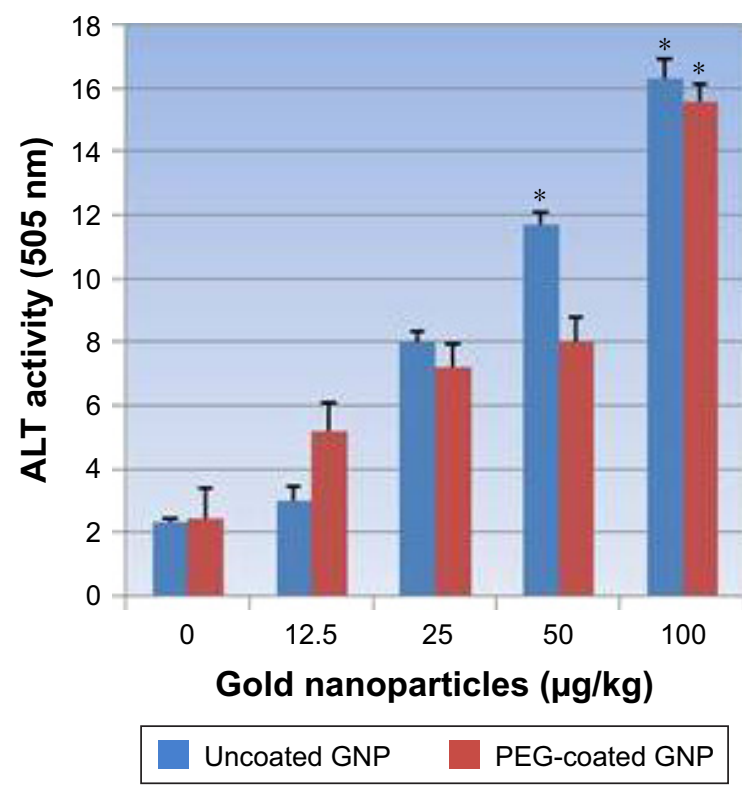

Figure 5 Effects of PEG-coated and uncoated gold nanoparticles on the activity of alanine aminotransferases.

Notes: The mean \pm SD of three rats represents each bar. Values shown with asterisks are significantly different from the control. $* P<0.05$.

Abbreviations: GNP, gold nanoparticle; PEG, poly-ethylene-glycol; ALT, alanine aminotransferase.

observed in uncoated GNPs than in PEG-coated GNPs relative to that of the negative control.

\section{Alanine aminotransferase}

As shown in Figure 5, we observed a dose-dependent increase in alanine activity (ALT/GPT) in PEG-coated and uncoated GNPs relative to the negative control. However, two high level doses, 50 and $100 \mu \mathrm{g} / \mathrm{kg}$, produced a statistically significant outcome in elevating the activity of ALT/GPT relative to the negative control. ALT activity was elevated to a greater extent in uncoated GNPs than in PEG-coated GNPs relative to the negative control.

\section{Aspartate aminotransferases}

PEG-coated and uncoated GNP exposure in rats increased AST/GOT activity levels relative to the negative control. Figure 6 shows experimental data acquired from assays of AST/GOT activity. As shown in the figure, the highest doses of $50 \mu \mathrm{g} / \mathrm{kg}$ and $100 \mu \mathrm{g} / \mathrm{kg}$ presented a statistically notable variation in enhancing the activity of AST/GOT in both types of GNPs relative to the negative control. However, uncoated GNP responded more strongly than PEG-coated GNPs.

\section{Alkaline phosphatases}

Figure 7 reflects the activity of ALP exposed to PEG-coated and uncoated GNPs. An elevation in the activity of ALPs in 


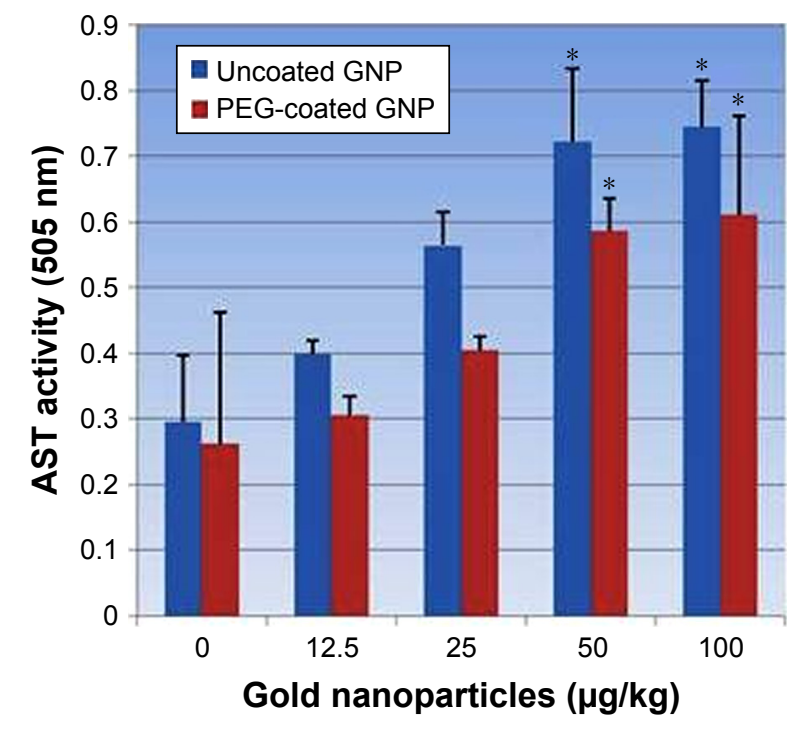

Figure 6 Effects of PEG-coated and uncoated gold nanoparticle on the activity of aspartate aminotransferases.

Notes: Mean \pm SD values for three rats represents each bar. Values shown with asterisks are significantly different from control. $* P<0.05$.

Abbreviations: GNP, gold nanoparticle; PEG, poly-ethylene-glycol; AST, aspartate aminotransferase.

rats treated with both types of GNPs relative to the negative control is shown in Figure 7. However, three concentrations $(25,50$, and $100 \mu \mathrm{g} / \mathrm{kg})$ showed statistically significant effects in elevating ALP activity in both PEG-coated and uncoated GNPs relative to the negative control. ALP activity was elevated more in uncoated GNPs than in PEG-coated GNPs.

\section{Histopathological evaluation}

The toxicity of PEG-coated and uncoated GNPs was examined via $\mathrm{H} \& \mathrm{E}$ staining. A histological evaluation of liver

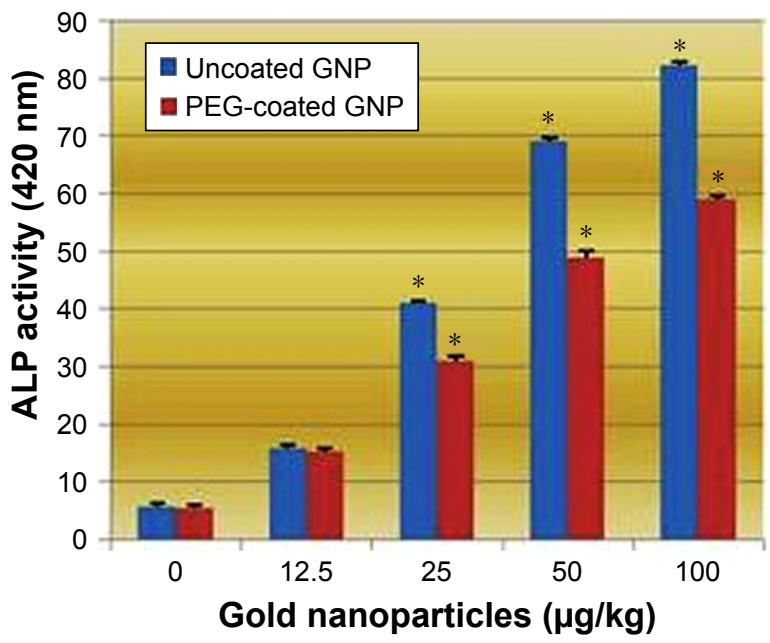

Figure 7 Effects of PEG-coated and uncoated gold nanoparticles on the activity of alkaline phosphatase.

Notes: The mean \pm SD of three rats represents each bar. Values shown with asterisks are significantly different from the control. $* P<0.05$.

Abbreviations: GNP, gold nanoparticle; PEG, poly-ethylene-glycol; ALP, alkaline phosphatise. samples exposed to either type of GNP did not reveal any evidence of inflammation. For the negative control group, a histological evaluation revealed a normal liver structure of compactly arranged hepatocytes. Sinusoids were randomly dispersed among hepatocytes and had consistent morphology along the central vein.

Rats dosed with $12.5,25,50$, and $100 \mu \mathrm{g} / \mathrm{kg}$ of PEGcoated and uncoated GNPs underwent unique morphological changes. Figure ( $8 \mathrm{Aa}$ ) is the photomicrograph of negative control (0) in rat livers exposed to uncoated GNPs. Damage to the central vein was observed through microscopic evaluations of rat livers exposed to $12.5 \mu \mathrm{g} / \mathrm{kg}$ (Figure $8 \mathrm{Ab}$ ) of uncoated GNP. The incomplete disturbance of central veins and vacuolation in hepatocytes (Figure $8 \mathrm{Ac}$ and $8 \mathrm{Bc}$, PEG-coated and uncoated GNPs) were observed in rat livers exposed to $25 \mu \mathrm{g} / \mathrm{kg}$ (Figure 8Ac) of uncoated GNP. In addition to the above-mentioned changes, liver degeneration (atrophy), injury to the central vein, and necrosis (Figure 8Ad, PEG-coated and uncoated GNPs) were observed in $50 \mu \mathrm{g} / \mathrm{kg}$ GNP exposed rat livers. In rat livers treated with $100 \mu \mathrm{g} / \mathrm{kg}$ of uncoated GNP, central vein impairment, necrosis (Figure 8Ae, uncoated GNP), and pyknotic or condensed nuclei (Figure 8Be, PEG-coated GNP) were observed. Figure $8 \mathrm{~A}$ shows photomicrographs of rat livers exposed to uncoated GNP and the negative control.

According to a microscopic examination of the PEGcoated exposed rats, liver samples showed less damage than the uncoated samples. Figure $8 \mathrm{Ba}$ shows the negative controls of rat livers exposed to PEG-coated GNP. In $12.5 \mu \mathrm{g} / \mathrm{kg}$ (Figure $8 \mathrm{Bb}$ ) PEG-coated livers, no morphological changes were observed, echoing results found for the negative control. Damage to the central vein was observed in $25 \mu \mathrm{g} / \mathrm{kg}$ (Figure 8Bc) exposed livers. In addition to the above-mentioned changes, vacuolation in hepatocytes and natural cell death were observed in $50 \mu \mathrm{g} / \mathrm{kg}$ (Figure 8Bd) GNP exposed rat livers. In $100 \mu \mathrm{g} / \mathrm{kg}$ (Figure $8 \mathrm{Be}$ ) group receiving PEG-coated GNPs, damage to the central vein, cell death, and pyknotic cells (Figure 8Be; PEG-coated GNP) were observed. Figure $8 \mathrm{~B}$ shows photomicrographs of rat livers exposed to PEG-coated GNP and the negative control.

However, uncoated GNPs underwent enhanced morphological changes relative to those of PEG-coated GNPs indicating that PEGylation may be used to improve the biocompatibility of GNPs used in a wide variety of biomedical applications.

\section{Discussion}

Comparative effects of PEG-coated and uncoated GNPsmediated hepatotoxicity and oxidative stress were examined 
A

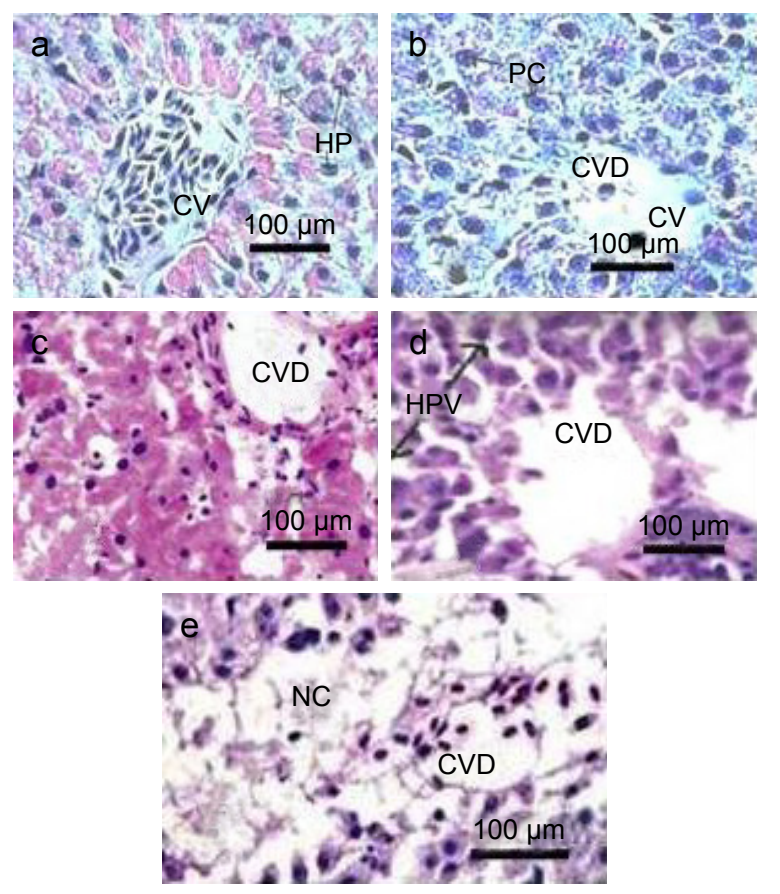

B

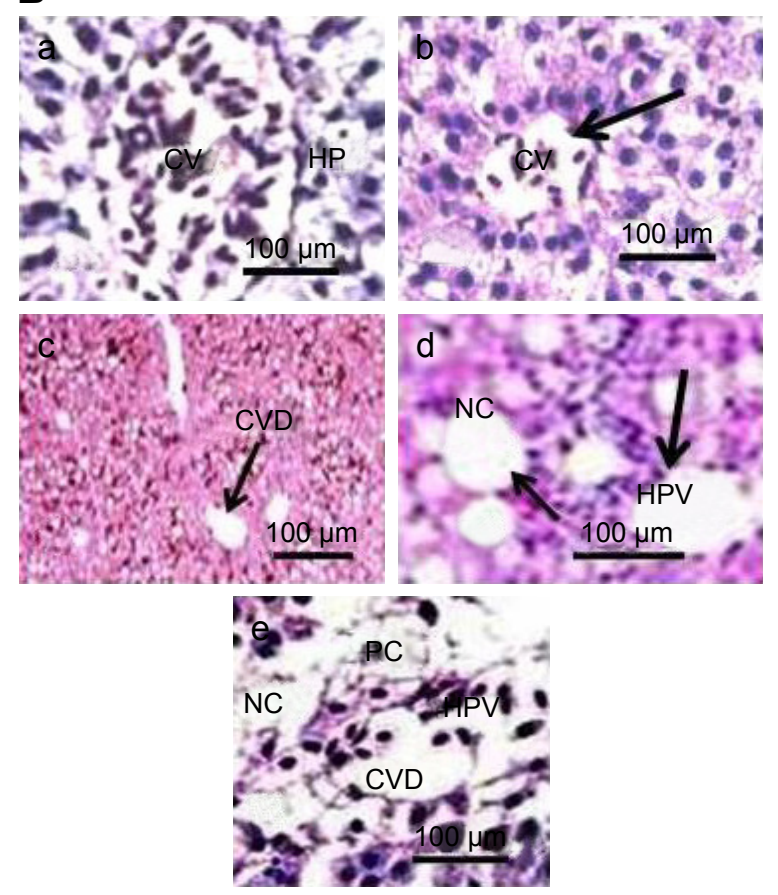

Figure 8 (A) Photomicrographs of the histology (H\&E staining, I,000X) of livers in Sprague Dawley rats exposed to uncoated gold nanoparticles. (a) Negative control (CV: central vein, HP: hepatocytes); (b) liver exposed tol $2.5 \mu \mathrm{g} / \mathrm{kg}$ (CV: central vein, CVD: damage to the central vein, PC: pycknotic); (c) liver exposed to $25 \mu \mathrm{g} / \mathrm{kg}$ (CVD: damage to the central vein, HPV: vacuolation in hepatocytes); (d) liver exposed to $50 \mu g / \mathrm{kg}$ (CVD: damage to the central vein, HPV: vacuolation in hepatocytes), and (e) liver exposed to $100 \mu \mathrm{g} / \mathrm{kg}$ (CVD: damage to the central vein, NC: necrosis). Mean values and standard deviations of ten values represent each point. (B) Histological photomicrographs (H\&E staining, I,000x) of the livers of Sprague Dawley rats exposed to PEG-coated gold nanoparticles. (a) Negative control (CV: central vein, HP: hepatocytes); (b) liver exposed to $12.5 \mu \mathrm{g} / \mathrm{kg}$ (CV: central vein); (c) liver exposed to $25 \mu \mathrm{g} / \mathrm{kg}$ (CVD: damage to the central vein); (d) liver exposed to $50 \mu \mathrm{g} / \mathrm{kg}$ (HPV: vacuolation in hepatocytes, NC: necrosis), and (e) liver exposed to $100 \mu g / k g$ (CVD: damage to the central vein, HPV: vacuolation in hepatocytes, NC: necrosis, PC: pyknotic). Mean values and standard deviations of ten values represents each point.

Note: Arrows indicate particular damage in the specimen.

in Sprague Dawley rats. Oxidative stress was determined by the amount of ROS produced; hepatotoxicity was characterized by ALT, AST, and ALP levels in serum; and from histopathological evaluations of liver samples.

In the liver, Kupffer cells play a significant role in its physiology and in maintaining equilibrium. Kupffer cells' activation by toxins results in the release of a series of inflammatory mediators, growth factors, and $\operatorname{ROS}^{18}$ that cause hepatocyte injury. To understand mechanisms of liver injury, we must understand the state of diverse responses displayed by Kupffer cells. ${ }^{19}$

Levels of ROS production observed in liver homogenates of all four experimental groups were analyzed by identifying oxidation-induced fluorescence emitted by the $\mathrm{H}_{2}$ DCFDA dye. The induction of ROS was elevated in rats exposed to PEG-coated and uncoated GNPs relative to the negative control. As reported in Figure 4, doses of 50 and $100 \mu \mathrm{g} / \mathrm{kg}$ GNPs applied to both types showed a statistically significant difference in the induction of ROS compared to the negative control. However, uncoated GNPs were shown to induce more ROS than PEG-coated GNPs. ROS has been found in many studies to produce an inflammatory state that promotes apoptosis across a diverse range of cell types. ${ }^{20,21}$
The best indicator for liver toxicity or damage is an elevation in serum ALT, AST, and ALP levels. These enzymes are contained in hepatocytes, and are released in the blood stream when cells are damaged. The biochemical measurement of these enzymes in serum assesses liver function failure, hepatocellular injury, and cholestasis. In our study, PEG-coated and uncoated GNP exposure resulted in an elevation of ALT and AST levels relative to the negative control. As is shown in Figures 5 and 6, doses of 50 and $100 \mu \mathrm{g} / \mathrm{kg}$ of both types of GNPs established a statistically significant difference in elevating ALT and AST activity relative to the negative control. However, uncoated GNP was shown to induce more damage than PEG-coated GNPs. In the cell lining of the liver, small bile ducts (ductoles) are responsible for ALP generation. ALP is the main enzyme that is known to elevate when liver disease is obstructive or cholestatic. In our study 25, 50, and $100 \mu \mathrm{g} / \mathrm{kg}$ doses of PEGcoated and uncoated GNPs exhibited a statistically significant difference in elevating ALP levels in serum compared to the negative control. Again, uncoated GNP was found to elevate ALP levels more than PEG-coated GNPs. Similar results have been reported by other investigators who found increased activity in these enzymes after GNP exposure. ${ }^{1,12,22}$ The enzyme 
activity in these studies was found to be much more higher than in our study, which may be due to the route of exposure.

Histopathological evaluations of livers exposed to PEG-coated and uncoated GNPs revealed significant morphological variations, such as damage to the central vein; vacuolation in hepatocytes (Figure 8Ac, uncoated GNP and 8D, PEG-coated GNP), pyknosis (Figure 8Be, PEGcoated GNP) or karyomegaly; or condensed hepatocytes' nuclei and necrosis (Figure 8Ae, uncoated GNP) compared to the negative control. The results of microscopic examinations of livers exposed to PEG-coated and uncoated GNPs are reported in Figure 8A and B. Both types of GNPs induced damage to the liver relative to the negative control. We found that uncoated GNP caused more damage than PEG-coated GNP. Corresponding outcomes have been published by Hwang et $\mathrm{al}^{12}$ as a result of studies of healthy and damaged livers of rodents during GNP-induced hepatotoxicity.

The modification of cell-particle interactions through the functionalization of GNP with ligands such as PEG (PEGylation) is known to stabilize blood components. The outcomes of our work are in agreement with Lipka et al's ${ }^{5}$ results which show a reduction in surface charges and an improvement in blood stability resulting from the PEGylation of GNPs. Since uncoated GNPs are susceptible to aggregation in solution due to high surface area to volume ratio and presence of ionic charges, both of which cause significant changes in their optical properties, uncoated GNPs can easily interact with biological molecules relative to PEG-coated GNPs, making it more damaging or toxic to cells and less stable. In addition to the functionalization of GNP, size is another remarkable modulator of in vivo toxicity. It has been reported that GNPs of size ranging from 5 to $30 \mathrm{~nm}$ enhance biochemical indicators; slight damage to the liver is caused by GNPs of 10-60 nm. ${ }^{23-27}$ PEG-coated GNPs of $13.5 \mathrm{~nm}$ have been found to accumulate in the liver and spleen. Additionally, GNPs of larger than $100 \mathrm{~nm}$ are released from the body, in part, through excretion. ${ }^{26}$ In our study, $14.5 \mathrm{~nm}$ PEG-coated GNPs and $22.5 \mathrm{~nm}$ uncoated GNPs increased the levels of biochemical markers of hepatotoxicity and caused damage to livers in the highest two concentrations (50 and $100 \mu \mathrm{g} / \mathrm{kg}$ ), as reported by a study ${ }^{23}$ where 5-30 nm GNPs were observed to produce similar effects.

\section{Conclusion}

In the present work, we have systematically studied the in vivo acute toxicity of PEG-coated and uncoated GNPs after oral administration. Our results show that uncoated GNP exposure spurs more biochemical serum changes, and ROS induction and injury to liver tissue than PEG-coated GNPs.
Functionalization and GNP size are two factors responsible for the outcomes of our study. Our results demonstrate that PEG-coated GNPs are safer to use in nanomedicinal applications than uncoated GNPs. However, more studies must confirm the outcomes of PEGylation. Currently, there is limited knowledge relating to their environmental toxicity and biological safety.

\section{Acknowledgments}

This research was funded by the National Institutes of Health (Grant \# G12MD007581) through the RCMI Center for Environmental Health.

\section{Author contributions}

$\mathrm{AKP}, \mathrm{PhD}$, performed most of the work by designing and performing experiments, collecting and analyzing data, and writing the manuscript; $\mathrm{SAK}, \mathrm{PhD}$, performed histological studies of liver tissues; and PBT, ScD, checked the project's specifications prior to starting experiments, proofread the manuscript, and provided financial support. All authors contributed toward data analysis, drafting and critically revising the paper, gave final approval of the version to be published and agree to be accountable for all aspects of the work.

\section{Disclosure}

The authors report no conflicts of interest in this work.

\section{References}

1. Chen J, Wang H, Long W, et al. Sex differences in the toxicity of polyethylene glycol-coated gold nanoparticles in mice. Int J Nanomedicine. 2013;8:2409-2419.

2. Yah CS. The toxicity of gold nanoparticles in relation to their physiochemical properties. Biomed Res. 2013;24(3):400-413.

3. Chithrani DB, Jelveh S, Jalali F, et al. Gold nanoparticles as radiation sensitizers in cancer therapy. Radiat Res. 2010;173(6):719-728.

4. Niidome T, Yamagata M, Okamoto Y, et al. PEG-modified gold nanorods with a stealth character for in vivo applications. $J$ Control Release. 2006;114(3):343-347.

5. Lipka J, Semmler-Behnke M, Sperling RA, et al. Biodistribution of PEG-modified gold nanoparticles following intratracheal instillation and intravenous injection. Biomaterials. 2010;31(25):6574-6581.

6. Wang A, Pu K, Dong B, et al. Role of surface charge and oxidative stress in cytotoxicity and genotoxicity of graphene oxide towards human lung fibroblast cells. J Appl Toxicol. 2013;33(10):1156-1164.

7. Sarkar A, Ghosh M, Sil PC. Nanotoxicity: oxidative stress mediated toxicity of metal and metal oxide nanoparticles. J Nanosci Nanotechnol. 2014;14(1):730-743.

8. Risom L, Møller P, Loft S. Oxidative stress-induced DNA damage by particulate air pollution. Mutat Res. 2005;592(1-2):119-137.

9. Nel A, Xia T, Mädler L, Li N. Toxic potential of materials at the nanolevel. Science. 2006;311(5761):622-627.

10. Misawa M, Takahashi J. Generation of reactive oxygen species induced by gold nanoparticles under $\mathrm{x}$-ray and UV irradiations. Nanomedicine: Nanotechnology. Biology and Medicine. 2011;7(5):604-614.

11. Limbach LK, Wick P, Manser P, Grass RN, Bruinink A, Stark WJ. Exposure of engineered nanoparticles to human lung epithelial cells: influence of chemical composition and catalytic activity on oxidative stress. Environ Sci Technol. 2007;41(11):4158-4163. 
12. Hwang JH, Kim SJ, Kim YH, et al. Susceptibility to gold nanoparticleinduced hepatotoxicity is enhanced in a mouse model of nonalcoholic steatohepatitis. Toxicology. 2012;294(1):27-35.

13. Seeff Z. Enzymes in hepatic disease. In: Goodly EL. editor. Diagnostic Enzymology. Philadelphia, PA: Lea \& Febiger; 1970:1-38.

14. Giles AR. Guidelines for the use of animals in biomedical research. Thromb Haemost. 1987;58(4):1078-1084.

15. Lawler J, Song W, Demaree SR. Hindlimb unloading increases oxidative stress and disrupts antioxidant capacity in skeletal muscle. Free Radic Biol Med. 2003;35(1):9-16.

16. Reitman S, Frankel S. A colorimetric method for the determination of serum glutamic oxalacetic and glutamic pyruvic transaminases. Am J Clin Pathol. 1957;28(1):56-63.

17. Kay HD. In Method of determination. Some properties of the enzyme. Plasma phosphatase. J Biol Chem. 1930;89:235.

18. Bilzer M, Jaeschke H, Vollmar AM, Paumgartner G, Gerbes AL. Prevention of Kupffer cell-induced oxidant injury in rat liver by atrial natriuretic peptide. Am J Physiol. 1999;276(5):G1137-G1144.

19. Roberts RA, Ganey PE, Ju C, Kamendulis LM, Rusyn I, Klaunig JE. Role of the Kupffer cell in mediating hepatic toxicity and carcinogenesis. Toxicol Sci. 2007;96(1):2-15.

20. Herrera B, Álvarez AM, Sánchez A, et al. Reactive oxygen species (ROS) mediates the mitochondrial-dependent apoptosis induced by transforming growth factor (beta) in fetal hepatocytes. Faseb J. 2001; 15(3):741-751.
21. Simon HU, Haj-Yehia A, Levi-Schaffer F. Role of reactive oxygen species (ROS) in apoptosis induction. Apoptosis. 2000;5(5):415-418.

22. Abdelhalim M, Moussa S, Qaid $\mathrm{H}$. The protective role of quercetin and arginine on gold nanoparticles induced hepatotoxicity in rats. Int J of Nanomedicine. 2018;13:2821-2825.

23. Zhang X-D, Wu D, Shen X, et al. Size-dependent radiosensitization of PEG-coated gold nanoparticles for cancer radiation therapy. Biomaterials. 2012;33(27):6408-6419.

24. de Jong WH, Hagens WI, Krystek P, Burger MC, Sips AJ, Geertsma RE. Particle size-dependent organ distribution of gold nanoparticles after intravenous administration. Biomaterials. 2008;29(12): 1912-1919.

25. Zhang XD, Wu D, Shen X, Chen J, et al. Size-dependent in vivo toxicity of PEG-coated gold nanoparticles. Int J Nanomedicine. 2011;6:2071-2081.

26. Cho WS, Cho M, Jeong J, et al. Size-dependent tissue kinetics of PEG-coated gold nanoparticles. Toxicol Appl Pharmacol. 2010;245(1): 116-123.

27. Balasubramanian SK, Jittiwat J, Manikandan J, Ong CN, Yu LE, Ong WY. Biodistribution of gold nanoparticles and gene expression changes in the liver and spleen after intravenous administration in rats. Biomaterials. 2010;31(8):2034-2042.

28. Guillen J. FELASA guidelines and recommendations. J Am Assoc Lab Anim Sci. 2012;51(3):311-321.
International Journal of Nanomedicine

\section{Publish your work in this journal}

The International Journal of Nanomedicine is an international, peerreviewed journal focusing on the application of nanotechnology in diagnostics, therapeutics, and drug delivery systems throughout the biomedical field. This journal is indexed on PubMed Central, MedLine, CAS, SciSearch $\AA$, Current Contents $₫ /$ Clinical Medicine,

\section{Dovepress}

Journal Citation Reports/Science Edition, EMBase, Scopus and the Elsevier Bibliographic databases. The manuscript management system is completely online and includes a very quick and fair peer-review system, which is all easy to use. Visit http://www.dovepress.com/ testimonials.php to read real quotes from published authors. 